Pacific Journal of Mathematics

ON THE THEORY OF HOMOGENEOUS LIPCHITZ SPACES 


\title{
ON THE THEORY OF HOMOGENEOUS LIPSCHITZ SPACES AND CAMPANATO SPACES
}

\author{
HARVEY GreENWALD
}

\begin{abstract}
In this paper the equivalence between the Campanato spaces and homogeneous Lipschitz spaces is shown through the use of elementary and constructive means. These Lipschitz spaces can be defined in terms of derivatives as well as differences.
\end{abstract}

Introduction. The Campanato spaces have previously been stated by Taibleson and Weiss [13] to be duals of certain Hardy spaces. Further results will be forthcoming in a paper by Janson, Taibleson, and Weiss.

For $k>\alpha$ we define,

$$
\Lambda_{\alpha, k}=\left\{[f] \in S^{\prime} / P_{k-1}: \sum_{|\nu|=k} \sup _{t \in \mathbf{R}^{+}} \sup _{x \in \mathbf{R}^{n}} t^{(k-\alpha) / 2}\left|D^{\nu} f(x, t)\right|<\infty\right\}
$$

where $f(x, t)$ is the Gauss-Weierstrass integral of $f$ and $D^{\nu} f(x, t)$ involves the derivatives with respect to the $x_{i}$.

Consider the collection of locally integrable functions $f$ for which

$$
\|f\|_{L(\alpha, p, k-1)}=\sup _{Q \subset \mathbf{R}^{n}}|Q|^{-\alpha / n}\left[\frac{1}{|Q|} \int_{Q}\left|f(x)-P_{Q} f(x)\right|^{p} d x\right]^{1 / p}<\infty
$$

where $Q$ is a ball, $k>\alpha$, and $P_{Q} f$ is the minimizing polynomial of degree $\leq(k-1)$. The space $L(\alpha, p, k-1)$ is defined as a space of equivalence classes modulo the polynomials of degree $\leq(k-1)$.

The principal result is the following.

Proposition. For $k>\alpha$, the following are equivalent spaces:

(i) $L(\alpha, p, k-1)$ and

(ii) $\Lambda_{\alpha, k}$.

In addition it is shown that the distributions in $\Lambda_{\alpha, k}$ are in fact slowly increasing functions.

Homogeneous Lipschitz spaces have been studied extensively by a number of people. The reader is referred, in particular, to Herz [5], Johnson [7], and Janson [6]. Grevholm [4] has proved that $L(\alpha, p, k-1)$ 
is equivalent to a space whose elements are in an interpolation space and whose norm is defined in terms of differences. Grevholm's proof is limited to $p<\infty$ and is done through the use of interpolation theory. The results in this paper are proved by elementary methods and are valid for $1 \leq p \leq \infty$.

The reader is assumed to be familiar with the standard properties of the Weierstrass kernel. A discussion of these properties can be found in Taibleson [12] and in Flett [3].

All immaterial constants shall be denoted by the same letter $C$.

Before beginning I would like to thank Mitchell Taibleson for his many suggestions concerning the material in this paper.

\section{Principal Results.}

Definition. Let $k>\alpha>0$ and let $P_{k-1}$ be the set of polynomials of degree $\leq(k-1)$. Define

$$
\begin{aligned}
\Lambda_{\alpha, k}=\left\{[f] \in S^{\prime} / P_{k-1}:\right. & \\
& \left.\|f\|_{\alpha, k}=\sum_{|\nu|=k} \sup _{t \in \mathbf{R}^{+}} \sup _{x \in \mathbf{R}^{n}} t^{(k-\alpha) / 2}\left|D^{\nu} f(x, t)\right|<\infty\right\}
\end{aligned}
$$

where $f \in[f], S^{\prime}$ is the set of tempered distributions, and

$$
D^{\nu} f(x, t)=\frac{\partial^{k}}{\partial x_{1}^{\nu_{1}} \cdots \partial x_{n}^{\nu_{n}}} f(x, t)
$$

for $\nu=\left(\nu_{1}, \ldots, \nu_{n}\right)$ and $|\nu|=\nu_{1}+\cdots+\nu_{n}=k$. Here $f(x, t)=f^{*} W(x, t)$ is the Weierstrass integral of $f$ where $W(x, t)=c t^{-n / 2} e^{-|x|^{2} / 4 t}$ is the Weierstrass kernel. See Taibleson [12] and Flett [3] for a discussion of Weierstrass integrals of distributions.

Lipschitz conditions on $S^{\prime} / P_{k-1}$ have been considered by Janson [6] and Johnson [7]. It is easy to check that $\|f\|_{\alpha, k}$ is independent of the coset respresentative and does indeed define a norm.

Definition. Consider the collection of locally integrable functions $f$ for which

$$
\|f\|_{L(\alpha, p, k-1)}=\sup _{Q \subset \mathbf{R}^{n}}|Q|^{-\alpha / n}\left[\frac{1}{|Q|} \int_{Q}\left|f(x)-P_{Q} f(x)\right|^{p} d x\right]^{1 / p}<\infty
$$


where $Q$ is a ball, $k>\alpha$, and where $P_{Q} f$ is the unique polynomial of degree $\leq(k-1)$ such that

$$
\int_{Q}\left[f(x)-P_{Q} f(x)\right] x^{\nu} d x=0
$$

for $0 \leq|\nu| \leq k-1$. The space $L(\alpha, p, k-1)$ is defined as a space of equivalence classes of these functions modulo $P_{k-1}$. See Taibleson and Weiss [13] for a complete discussion of these notions.

Proposition 1. Let $[f] \in L(\alpha, p, k-1)$. Then $[f] \in \Lambda_{\alpha, k}$ and $\|f\|_{\alpha, k} \leq C\|f\|_{L(\alpha, p, k-1)}$.

Proof. Let $[f] \in L(\alpha, p, k-1)$ and $f \in[f]$. Then

$$
f(x)[1+|x|]^{-n / p-\varepsilon} \in L^{p} \text { for } \varepsilon>\max (k, \alpha) .
$$

See Ricci and Taibleson [10]. Hence $f \in S^{\prime}$.

Now let $x_{0} \in \mathbf{R}^{n}, t \in \mathbf{R}^{+}, Q=B_{x_{0}}\left(t^{1 / 2}\right)$ be the ball about $x_{0}$ of radius $t^{1 / 2}$, and $P=P_{Q}$. Then $P(x, t)$ is also a polynomial of degree $\leq(k-1)$. If $|\nu|=k$,

$$
\begin{aligned}
& D^{\nu} f\left(x_{0}, t\right)=D^{\nu} f\left(x_{0}, t\right)-D^{\nu} P\left(x_{0}, t\right) \\
& =\int_{\mathbf{R}^{n}}[f(y)-P(y)] D^{\nu} W\left(x_{0}-y, t\right) d y \\
& =\int_{\mathbf{R}^{n}} \frac{[f(y)-P(y)] t^{\varepsilon / 2}}{\left[t^{1 / 2}+\left|y-x_{0}\right|\right]^{n / p+\varepsilon}} \frac{\left[t^{1 / 2}+\left|y-x_{0}\right|\right]^{n / p+\varepsilon}}{t^{\varepsilon / 2}} D^{\nu} W\left(x_{0}-y, t\right) d y .
\end{aligned}
$$

Apply Hölder's inequality.

$$
\left\|\frac{[f(y)-P(y)] t^{\varepsilon / 2}}{\left[t^{1 / 2}+\left|y-x_{0}\right|\right]^{n / p+\varepsilon}}\right\|_{P} \leq C\|f\|_{L(\alpha, p, k-1)} t^{\alpha / 2} .
$$

See Ricci and Taibleson.

(b)

$$
\left\|\frac{\left[t^{1 / 2}+\left|y-x_{0}\right|\right]^{n / p+\varepsilon}}{t^{\varepsilon / 2}} D^{\nu} W\left(x_{0}-y, y\right)\right\|_{P^{\prime}} \leq I_{1}+I_{2}
$$

where the first integration is over $\left|y-x_{0}\right| \leq t^{1 / 2}$ and the second is over $\left|y-x_{0}\right|>t^{1 / 2}$. 
(i)

$$
\begin{aligned}
I & \leq C\left(\int_{|y| \leq t^{1 / 2}}\left[t^{n / 2 P+\varepsilon / 2} t^{-\varepsilon / 2}\left|D^{\nu} W(y, t)\right|\right]^{P^{\prime}} d y\right)^{1 / P^{\prime}} \\
& \leq C t^{n / 2} t^{-k / 2} t^{-n / 2}=C t^{-k / 2}
\end{aligned}
$$

by changing variables and using the usual estimates for $W(y, t)$.

(ii)

$$
\begin{aligned}
I_{2} & \leq C\left(\int_{|y|>t^{1 / 2}}\left[\frac{|y|^{n / p+\varepsilon}}{t^{\varepsilon / 2}}\left|D^{\nu} W(y, t)\right|\right]^{P^{\prime}} d y\right)^{1 / P^{\prime}} \\
& \leq C\left(\int_{|\mu| \geq 1}\left[t^{n / 2 P+\varepsilon / 2}|\mu|^{n / p+\varepsilon} t^{-\varepsilon / 2} t^{-k / 2}|\mu|^{k} t^{-n / 2} e^{-|\mu|^{2} / 4}\right]^{P^{\prime}} t^{n / 2} d \mu\right)^{1 / P^{\prime}}
\end{aligned}
$$

where $\mu=y t^{-1 / 2}$. The above is bounded by

$$
C t^{n / 2 P-k / 2-n / 2+n / 2 P^{\prime}}=C t^{-k / 2} \text {. }
$$

(c) Hence

$$
\sup _{x_{0} \in \mathbf{R}^{n}}\left|D^{\nu} f\left(x_{0}, t\right)\right| \leq C\|f\|_{L(\alpha, p, \beta-1)} t^{\alpha / 2} t^{-k / 2}
$$

and the result follows.

Definition. Let $\Delta(h) f(x)=f(x-h)-f(x)$. For $k>\alpha$ we define $A_{\alpha, k}\left\{[f] \in S^{\prime} / P_{k-1}\right.$ :

$$
\begin{aligned}
\|f\|_{A_{\alpha, k}}=\sup _{h_{1}, \ldots, h_{k} \in \mathbf{R}^{n}} \sup _{x \in \mathbf{R}^{n}}\left[\left|h_{1}\right|+\cdots+\left|h_{k}\right|\right]^{-\alpha} \\
\left.\cdot\left|\Delta\left(h_{1}\right) \cdots \Delta\left(h_{k}\right) f(x)\right|<\infty\right\}
\end{aligned}
$$

where $\Delta\left(h_{1}\right) \cdots \Delta\left(h_{k}\right) f(x)$ is defined in the distribution sense and is assumed to be an $L^{\infty}$ function. Spaces similar to this have been studied by Janson [6].

LEMMA 1. For $k>\alpha$, the following are equivalent spaces:

(i) $\Lambda_{\alpha, k}$ and

(ii) $A_{\alpha, k}$.

Proof. This can be proved by standard arguments. See Johnson [7] or Janson [6] for similar results. 
LEMMA 2. Let $[f] \in \Lambda_{\alpha, k}$ and let $f$ be a slowly increasing function. Then $[f] \in L(\alpha, p, k-1)$ and

$$
\|f\|_{L(\alpha, p, k-1)} \leq C\|f\|_{\alpha, k} .
$$

Proof. Since $[f] \in \Lambda_{\alpha, k}$,

$$
\left|\Delta\left(h_{1}\right) \cdots \Delta\left(h_{k}\right) f(x)\right| \leq C\|f\|_{\alpha, k}\left[\left|h_{1}\right|+\cdots+\left|h_{k}\right|\right]^{\alpha}
$$

for every $h_{1}, \ldots, h_{k} \in \mathbf{R}^{n}$ by Lemma 1 . We shall show that $f \in$ $L(\alpha, \infty, k-1)$. Let $x_{0} \in \mathbf{R}^{n}, \delta \in \mathbf{R}^{+}$, and $Q=B_{x_{0}}(\delta)$. We shall find a polynomial $P$ of degree $\leq(k-1)$ such that

$$
\sup _{x \in Q}|f(x)-P(x)| \leq C\|f\|_{\alpha, k} \delta^{\alpha} .
$$

The result will then easily follow.

(a) Using an argument of Janson [6] (Theorem 6), we see that

$$
\begin{aligned}
& (-1)^{k-1} \int \cdots \int \Delta\left(h_{1}\right) \cdots \Delta\left(h_{k}\right) f(x) W\left(h_{1}, t\right) \\
& \cdots W\left(h_{k}, t\right) d h_{1} \cdots d h_{k}=G(x, t)-f(x)
\end{aligned}
$$

where

$$
G(x, t)=\sum_{i=1}^{k} C_{i} f(x, i t) \text { and } C_{i}=(-1)^{i-1}\left(\begin{array}{l}
k \\
i
\end{array}\right) .
$$

It easily follows from the above that

$$
|G(x, t)-f(x)| \leq C\|f\|_{\alpha, k} t^{\alpha / 2} .
$$

(b) Let $t=\delta^{2}$. Then

$$
\left|G\left(x, \delta^{2}\right)-f(x)\right| \leq C\|f\|_{\alpha, k} \delta^{2}
$$

and

$$
G\left(x, \delta^{2}\right)=\sum_{i=1}^{k} C_{i} f\left(x, i \delta^{2}\right) .
$$

(c) Let $P\left(x, i \delta^{2}\right)$ be the Taylor polynomial about $\left(x-x_{0}\right)$ of degree $(k-1)$ for $f\left(x, i \delta^{2}\right)$. Then

$$
\begin{aligned}
\mid f\left(x, i \delta^{2}\right)- & P\left(x, i \delta^{2}\right)\left|\leq C \sum_{|\nu|=k}\right| D^{\nu} f\left(y_{\nu}, i \delta^{2}\right)|| x-\left.x_{0}\right|^{k} \\
& \leq C\|f\|_{\alpha, k}\left(i \delta^{2}\right)^{\alpha / 2-k / 2} \delta^{k} \\
& \leq C\|f\|_{\alpha, k} \delta^{\alpha} \quad \text { if } x \in B_{x_{0}}(\delta) .
\end{aligned}
$$


(d) Let $P(x)=\sum_{i=1}^{k} C_{l} P\left(x, i \delta^{2}\right)$. Then

$$
\begin{aligned}
|f(x)-P(x)| & \leq\left|f(x)-G\left(x, \delta^{2}\right)\right|+\left|G\left(x, \delta^{2}\right)-P(x)\right| \\
& \leq C\|f\|_{\alpha, k} \delta^{\alpha} .
\end{aligned}
$$

Proposition 2. Let $[f] \in \Lambda_{\alpha, k}, k>\alpha$, and $f \in[f]$. Then

(a) $f$ is a slowly increasing function,

(b) $[f] \in L(\alpha, p, k-1)$, and

(c) $\|f\|_{L(\alpha, p, k-1)} \leq C\|f\|_{\alpha, k}$.

Proof. Parts (b) and (c) follow immediately from part (a) and Lemma 2. Hence it suffices to prove (a). Let $f(x, s)$ be the Gauss-Weierstrass integral of $f$. Since $f(x, s)$ is slowly increasing,

$$
\begin{gathered}
(-1)^{k} \int \cdots \int \Delta\left(h_{1}\right) \cdots \Delta\left(h_{k}\right) f(x, s) W\left(h_{1}, t\right) \cdots W\left(h_{k}, t\right) d h_{1} \cdots d h_{k} \\
+\sum_{i=1}^{k} C_{l} f(x, i t+s)=f(x, s)
\end{gathered}
$$

as in Lemma 2. We claim that the limit as $s \rightarrow 0$ exists. This is immediate for the term involving $f(x, i t+s)$ since $f(x, i t+s)$ is continuous. For the first term, note that $\Delta\left(h_{1}\right) \cdots \Delta\left(h_{k}\right) f(x, s)$ is the Gauss-Weierstrass integral of the $L^{\infty}$ function $\Delta\left(h_{1}\right) \cdots \Delta\left(h_{k}\right) f(x)$ and hence converges for a.e. $x$. An easy application of the Dominated Convergence Theorem finishes the proof of the claim.

Let $g(x)=\lim _{s \rightarrow 0} f(x, s)$. Then

$$
\begin{aligned}
g(x)=(-1)^{k} \int & \cdots \int \Delta\left(h_{1}\right) \cdots \Delta\left(h_{k}\right) f(x) W\left(h_{1}, t\right) \\
& \cdots W\left(h_{k}, t\right) d h_{1} \cdots d h_{k}+\sum_{i=1}^{k} C_{l} f(x, i t) .
\end{aligned}
$$

The first term is a bounded function which is bounded by $C\|f\|_{\alpha, k} t^{\alpha / 2}$. The second term is slowly increasing since $f \in S^{\prime}$. Hence $g(x)$ is slowly increasing. Finally, we claim that $f=g$ as a distribution. A direct computation yields that $g(x, s)=f(x, s)$. Let $\phi \in S$. Then

$$
f(\phi)=\lim _{s \rightarrow 0} f(x, s)(\phi)=\lim _{s \rightarrow 0} g(x, s)(\phi)=g(\phi) .
$$

Note that for $f \in S^{\prime}$, the semi-group property of $f(x, t)$ and the fact that $f$ is slowly increasing as a function of $x$ follow from Flett [3]. 


\section{REFERENCES}

1. S. Campanato, Proprietà di hölderianità di alcune classi di funzioni, Ann. Scuola Norm. Sup. Pisa, (3) 17 (1963), 175-188.

2. R. R. Coifman and G. Weiss, Extensions of Hardy spaces and their use in analysis, Bull. Amer. Math. Soc., 83 (1977), 569-645.

3. T. M. Flett, Temperatures, Bessel potentials, and Lipschitz spaces, Proc. London Math. Soc., (3) 22 (1971), 385-451.

4. B. Grevholm, On the structure of the spaces $L_{k}^{p, \lambda}$, Math. Scand., 26 (1970), 241-254.

5. C. S. Herz, Lipschitz spaces and Bergstein's theorem on absolutely convergent Fourier transforms, J. Math. Mech., 18 (1968), 283-324.

6. S. Janson, Generalizations of Lipschitz spaces and an application to Hardy spaces and bounded mean oscillation, Duke Math. J., 47 (1980), 959-982.

7. R. Johnson, Temperatures, Riesz potentials, and the Lipschitz spaces of Herz, Proc. London Math. Soc., 27 (1973), 290-316.

8. B. H. Qui, Harmonic functions, Riesz potentials and the Lipschitz spaces of Herz, Hiroshima Math. J., 9 (1979), 245-295.

9. F. Ricci and M. Taibelson, Representation theorems for holomorphic and harmonic functions on mixed norm spaces, to appear.

10. Boundary values of harmonmic functions in mixed norm spaces and their atomic structure, to appear.

11. R. S. Strichartz, Bounded mean oscillation and Sobolev spaces, Indiana Univ. Math. J., 29 (1980), 539-558.

12. M. H. Taibleson, On the theory of Lipschitz spaces of distributions on Euclidean n-spaces, J. Math. Mech., 13 (1964), 407-480.

13. M. H. Taibleson and G. Weiss, The molecular characterization of certain Hardy spaces, Astérisque, 77 (1980), 68-149.

Received March 12, 1981 and in revised form December 11, 1981.

WASHINGTON UNIVERSITY

ST. LOUIS, MO 63130 



\section{PACIFIC JOURNAL OF MATHEMATICS EDITORS}

DONALD BABBITT (Managing Editor)

University of California

Los Angeles, CA 90024

Hugo Rossi

University of Utah

Salt Lake City, UT 84112

C. C. Moore and Arthur Ogus

University of California

Berkeley, CA 94720
J. DugunduI

Department of Mathematics

University of Southern California

Los Angeles, CA 90089-1113

R. FinN and H. SAmelson

Stanford University

Stanford, CA 94305

\section{ASSOCIATE EDITORS}
R. ARENS
E. F. BECKENBACH
B. H. NeUmaNN
F. WolF
K. YoshidA (1906-1982)

\section{SUPPORTING INSTITUTIONS}

UNIVERSITY OF ARIZONA

UNIVERSITY OF BRITISH COLUMBIA

CALIFORNIA INSTITUTE OF TECHNOLOGY

UNIVERSITY OF CALIFORNIA

MONTANA STATE UNIVERSITY

UNIVERSITY OF NEVADA, RENO

NEW MEXICO STATE UNIVERSITY

OREGON STATE UNIVERSITY
UNIVERSITY OF OREGON

UNIVERSITY OF SOUTHERN CALIFORNIA

STANFORD UNIVERSITY

UNIVERSITY OF HAWAII

UNIVERSITY OF TOKYO

UNIVERSITY OF UTAH

WASHINGTON STATE UNIVERSITY

UNIVERSITY OF WASHINGTON 


\section{Pacific Journal of Mathematics}

\section{Vol. 106, No. $1 \quad$ November, 1983}

John Ballard, Clifford's theorem for algebraic groups and Lie algebras . . . . . 1

Philip Richard Bartick, II and Edwin Duda, Finite-to-one open mappings

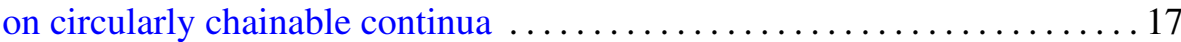

Frank Hayne Beatrous, Jr., $H^{\infty}$-interpolation from a subset of the

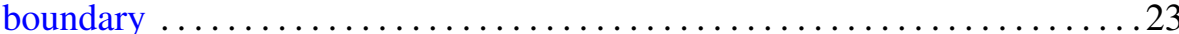

Sterling K. Berberian, Expectations in semifinite algebras ............ 33

Heron S. Collins and Wolfgang Ruess, Weak compactness in spaces of compact operators and of vector-valued functions $\ldots \ldots \ldots \ldots \ldots \ldots . \ldots 45$

David Downing and William O. Ray, Renorming and the theory of

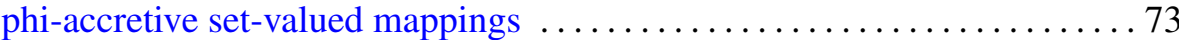

Harvey Charles Greenwald, On the theory of homogeneous Lipschitz

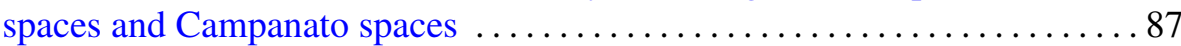

Irwen Valle Guadalupe and Lucio Ladislao Rodriguez, Normal curvature

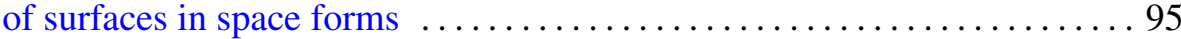

Boju Jiang, On the computations of the Nielsen number ............... 105

William H. Julian, Ray Mines, III and Fred Richman, Alexander

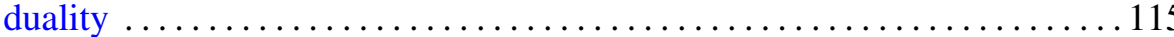

Midori Kobayashi, The connected component of the idèle class group of an

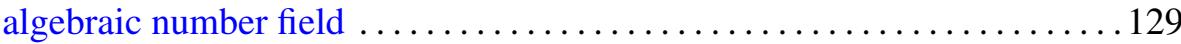

Ib Henning Madsen, Charles B. Thomas and C. Terence C. (Charles)

(Clegg) Wall, Topological spherical space form problem. III.

Dimensional bounds and smoothing

A. Sapounakis, The existence of strong liftings for totally ordered measure

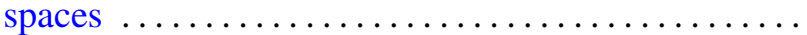

Gary Seitz, The root subgroups for maximal tori in finite groups of Lie type

Laurent Siebenmann and James M. Van Buskirk, Construction of irreducible homology 3-spheres with orientation reversing involution 